\title{
RE-DESIGN OF A MOTORCYCLE HELMET FOR USE IN URBAN TRAFFIC: CONCEPTUAL DESIGN AND TESTING
}

\author{
Papadopoulou, Joanna; \\ Papakostopoulos, Vassilis; \\ Moulianitis, Vassilis C. \\ University of the Aegean, Department of Product and Systems Design Engineering
}

\begin{abstract}
This paper presents the re-design approach of an urban motorcycle helmet to prevent users bypassing the strap fastening system. Related studies show that although a full-face helmet provides the maximum protection to a rider, in practice, full-face helmeted riders in urban traffic tend to improperly fasten it. On that notion, the design goal was to conceive a helmet that combines the advantages of different helmet types while responding to urban driving needs. During design ideation possible solutions were examined focusing on different ways of accessing and fixating the helmet on a rider's head, without using a strap fastening system. Preliminary concept development produced three design concepts, that were evaluated using two sets of prototypes: (a) the 3D printing method under a 1:2 scale was used to detect any design faults, while the 3D modeled concepts were evaluated in four different crash impacts regarding total deformation and von-Mises stress, and (b) 1:1 models of the three concepts were used by experienced riders to assess possible usability issues during helmet placement/removal. Results of the two-phase evaluation of the three concepts and design issues for further development of them are discussed.
\end{abstract}

Keywords: Conceptual design, Early design phases, User centred design, 3D printing, Functional modelling

\section{Contact:}

Papadopoulou, Joanna

University of the Aegean

Department of Product and Systems Design Engineering

Greece

jo.papdou@gmail.com 


\section{INTRODUCTION}

It is well established that head injuries are accounted for $70 \%$ of road accident deaths and most associated with non-helmet wearing motorcycle riders (World Health Organization, 2018; Lin and Kraus, 2009). However, helmeted riders involved in road accidents also suffer from severe facial injuries (Saunders et al, 2020; Kim et al, 2018; Ramli et al, 2014), which are related to improper use of helmet and helmet ejection especially in the case that the rider is thrown from the vehicle (Ramli and Oxley, 2016; Thai, McIntosh, and Pang, 2015; Yu et al., 2011).

Improper use of helmet is described by loose helmet fixation and/or retention fastening status that highly increases the riders' risk of suffering a traumatic brain injury or even death (Freitas et al, 2018; Jung, Xiao and Yoon, 2013). Factors that encourage either helmet non-use or improper helmet use are riders' discomfort, low-risk perception and insufficient traffic law enforcement among others (Li et al, 2008; Khan et al, 2008). More specifically, improper helmet use is more likely to be observed in short driving distances where frequent helmet placement and removal are required due to frequent stops (Hung, Stevenson and Ivers, 2008). Also, riders' exposure to lower risk because of short driving distances seems to favour helmet non-use or improper use, without necessarily implying a lower level of riders' subjective risk (see Ouellet, 2011; Esmaeilikia et al, 2019 for motorcycle and bicycle helmet use, respectively). Finally, road users' compliance with traffic law might be increased in the presence of law enforcement but only normative, e.g. compliance to the law requirement for helmet use without a clear determination of its fixation (Li et al, 2008; Kulanthayan et al, 2001; Skalkidou et al, 1999).

There are many types of helmets available in the market, however, three main types are most commonly observed among urban motorcycle riders, namely: full-face, open-face and half-face. Out of these three types, a full-face helmet is considered as the safest one, since it provides coverage to the entire head, including the chin area. However, a great many of riders in urban traffic tend to wear an open-face helmet or a half-face one, since the absence of chin protection and face coverage increases among others- the riders' convenience in terms of ease of helmet removal and placement (Ramli and Oxley, 2016; Yu et al, 2011; Tsai, Wang and Huang, 1995). In addition, other authors note that a number of full-face helmeted riders in urban traffic tend to improperly secure the retention system possibly because of feeling the helmet more stable on their head (Freitas et al, 2018).

Therefore, despite the recognised benefits of a full-face helmet in terms of safety, a certain barrier for full-face helmet wearing in urban traffic seems to be the effort needed by a rider for placing and fixing the helmet on his/her head properly compared to the number of repetitions required on a daily basis. To overcome this barrier, the objective of this paper is to explore design alternatives to eliminate the need of the typical strap fastening system, having a two-fold aim: (i) to reduce -if not to eliminate- the possibility of improper helmet fastening and fixing it on a rider's head and (ii) to facilitate the ease of helmet placement and removal. It is proposed that meeting the desired end of proper use of helmet in urban traffic can both maximize a rider's head protection and the effectiveness of traffic police enforcement.

The remaining of the paper is structured as follows: in section 2, design method and digital tools for the concept design development, prototyping and evaluation are presented; in section 3 , the proposed concept designs are evaluated both in terms of safety and ease of placement; finally, in section 4, the outcomes of the concepts' evaluation and directions for future development of them are discussed.

\section{DESIGN CONCEPT DEVELOPMENT}

A well-structured design approach should be elaborated in order to tackle the helmet design problem. Well-known design approaches such as the systematic approach framework (Pahl et al, 2007), the axiomatic design (Suh, 1990), the mechanical design process (Ullman, 2002) can be used among others.

In this paper, the problem-solving methodology, described by Dieter and Schmidt (2013), is used in order to satisfy new user needs by adjusting an already existing design solution through adaptive design. This methodology allows a designer to (i) define the problem and gather critical information, (ii) generate alternative and conceptual solutions, (iii) evaluate the alternatives and make decisions and (iv) communicate final details. 


\subsection{Problem definition}

Considering the advantages and disadvantages of a full-face and an open-face helmet, it is evident that each helmet type arrives at a different compromise between safety and ease of use. In particular, a fullface helmet offers the maximum possible safety to a rider, since it protects the entirety of the head including the chin area, that is the most common area to be injured in a crash and may lead to highly severe head injuries (Otte, 1991). However, this particular shell form has an adverse effect in terms of ease of use, since it contributes to the complexity of helmet placement/removal as well as to an increased helmet weight which is an important factor of riders' discomfort during riding. In contrast, an open-face helmet does not protect the chin area, but it is still considered almost as safe as a full-face helmet since it protects a very large area of the head (Sung et al, 2016; Yu et al, 2011). Due to the above compromise, this exact shell form makes it easier for the helmet to be placed /removed and also increases the likelihood to be properly fastened than a full-face helmet.

Therefore, the desired outcome of the design process (i.e. the main design challenges) is to propose a concept that offers simultaneously:

- maximum possible safety in terms of form (i.e. protecting the entirety of the head) and resistance in a crash (i.e. deformation of the shell remains below $6 \mathrm{~mm}$ for a $5 \mathrm{~kg}$ objects with $3.5 \mathrm{~m} / \mathrm{s}$ velocity, according to the European minimum safety requirements of a motorcycle helmet, namely ECE 22.05),

- ease of use during helmet placement/removal, and

- a minimum weight (aiming for less than $2000 \mathrm{gr}$ in consistence with helmets of the market).

\subsection{Design ideation}

During this phase, it is important to produce multiple ideas to find solutions to the aforementioned design challenges. The ideation is focused mainly on different ways to access the inner side of the helmet, i.e. how to open/close it. To better serve this direction, multiple locking mechanisms are examined so as to prevent users bypassing the strap fastening system. As a result, the design direction is led towards the removal of the typical strap fastening system by introducing a certain design feature, i.e. a solid body shell form under the chin, that eliminates the need for a fastening strap.

Based on this design feature, multiple ideas are produced in search for various solutions for enabling the head passing through a helmet aperture and the fastening of the helmet. Illustrative examples of different shell form variations combined with easy-to-use locking systems are seen in Figure 1.

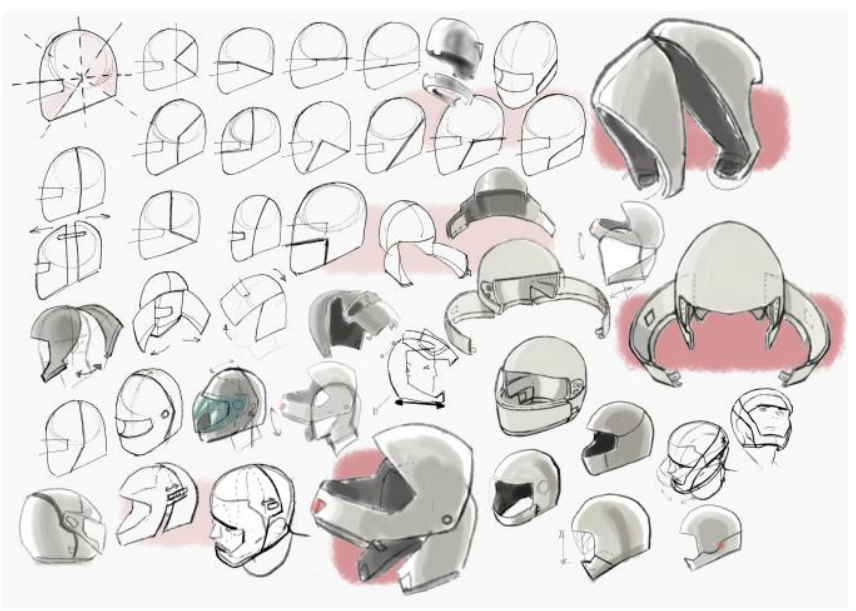

Figure 1. Ideation for the access and fastening of the helmet

\subsection{Preliminary concept development}

Following the previous phase, three different design directions emerge. As it is seen in Figure 2, each concept has a different way to open/close and secure. More specifically, the main direction behind the first concept (hereafter named as "Elytra") is to keep the shell form of an "open-face" helmet, allowing for fastening it through the front area of the head, i.e. around the chin. Accordingly, the idea behind the second concept (hereafter named as "Pacman") is to keep as much of the "full-face" shell intact, allowing for rear access of the head to the inside of the helmet in a way that resembles a hug-like 
closure around the head. Finally, the third concept (hereafter named as "Hood") is mainly driven by the so-called "flip-up" helmet and inspired by the familiar kinesiology for wearing a baseball hat.

Therefore, all three concepts meet the design criterion of protecting the entirety of the head, if locked, but in each concept a different articulation of the individual elements is used, namely: "Elytra" has a large solid body and a smaller one that are intersected at the chin area; "Pacman" has two solid bodies that are intersected diagonally on the horizontal axis, whereas, "Hood" has two solid bodies where one rotated out of the other on the vertical axis.

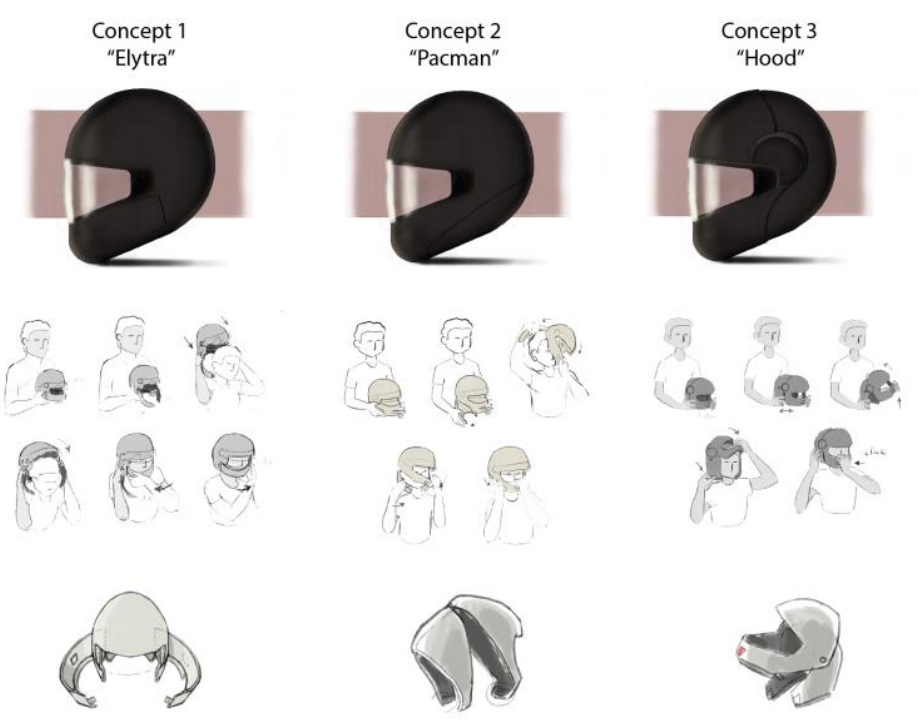

Figure 2. Concepts development

\subsection{Prototyping}

To ensure that each of the three concepts meets the design challenges, they are evaluated separately in terms of safety (i.e. testing helmet behaviour in simulated crash scenarios) and usability (i.e. ease of helmet placement/removal). To this end, two sets of prototypes are used.

The first set includes scaled 3D printed models of the shells facilitating the detection of potential faults in the design of each concept (Figure 3a). The models are built around the ECE 22.05 reference head of size G (Small) to facilitate prototyping. For the 3D modelling of the head, the geometry parameters provided by the ECE guidelines are used. Also, the helmets shells are designed so as to leave room for protective foams and comfortability gaps. Based on the observations made on the scaled 3D printed models, the required design changes are incorporated in the final 3D models that are used in the crash simulations (see section 3.1). Taking into account these changes, the total weight of the models is less than 2000 gr, satisfying the corresponding design criterion.

The second set of prototypes includes functional models of the three concepts that are made from scrapped helmets (Figure 3b). These models were used by experienced motorcycle riders to bring into the fore possible usability issues during helmet placement/removal (see section 3.2).

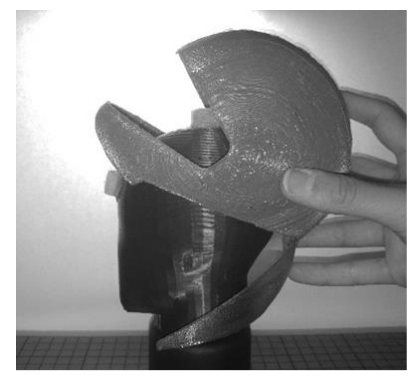

(a)

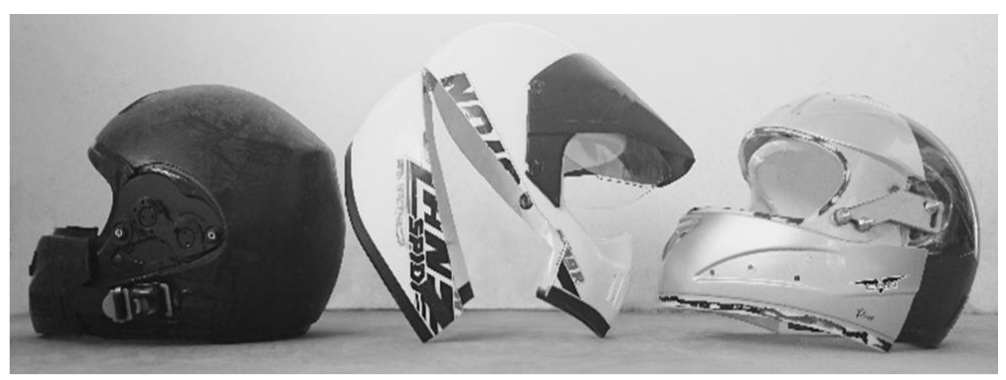

(b)

Figure 3. (a) Scaled 3D printed model of "Pacman", (b) Functional models of the 3 concepts 


\section{DESIGN CONCEPT EVALUATION}

The three design concepts are evaluated at two succeeding phases using the two sets of prototypes mentioned above. At evaluation phase 1, crash simulations are conducted to test the helmets' behaviour under different types of impact. At evaluation phase 2, a usability testing is conducted aiming to examine how well a particular concept fits the action capabilities of the user during helmet placement and removal.

\subsection{Evaluation phase 1: Impact simulations}

During this analysis, the 3D models of the three concepts' shells are used in the recreation of four different digital crash simulations, namely: front impact, rear impact, lateral impact and vertical impact.

\subsubsection{Simulation setup}

The models are created using the design programs Solidworks 2018 and Creo Parametric 5.0, while the explicit dynamics simulation is conducted using ANSYS Workbench 16.0. To better simulate the impact, a model of a barrier is constructed, as seen in Figure 4, and its lateral edges are fully fixed. The assigned material for the barrier is structural steel while for the helmet is ABS plastic. Both helmet and barrier are modelled using shell elements (8noded Shell 281). The interface between helmet and barrier is modelled using contact elements. The helmet has an initial impact velocity of 13 $\mathrm{m} / \mathrm{s}$ applied at the centre of mass along the axis of movement $(Z)$. Helmet displacement is set to 0 along $\mathrm{Y}$ axis, and free along $\mathrm{X}$ and $\mathrm{Z}$ axis.

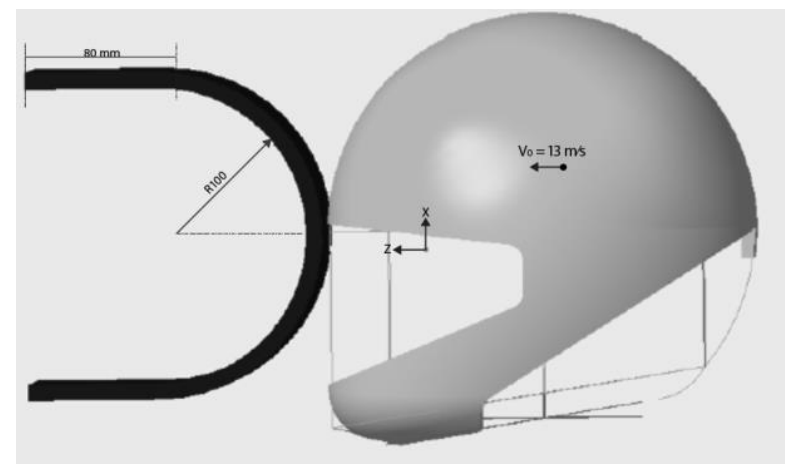

Figure 4. 3D models of the barrier and a helmet concept in Creo Parametric environment
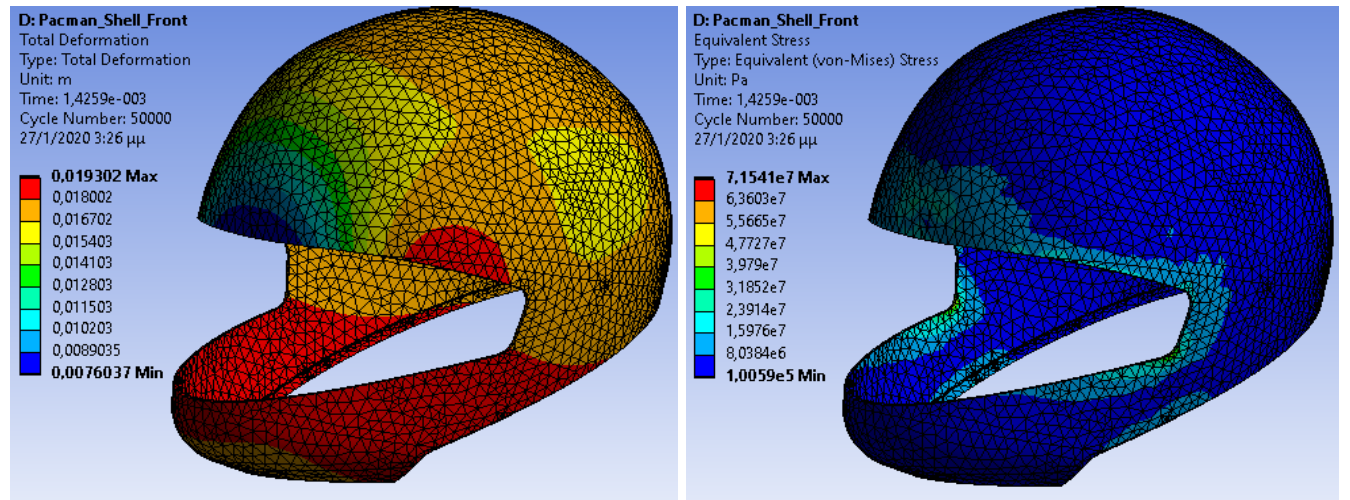

Figure 5. Total deformation and von-Mises stress of the Concept 2 in front impact.

The analyses ran for 50000 cycles simulating an impact of $0.01 \mathrm{sec}$ duration. To choose the best impact simulation duration, test analyses are made for $0 \mathrm{sec}, 0.01 \mathrm{sec}, 0.0001 \mathrm{sec}$ and $0.0007 \mathrm{sec}$ out of which for the last two, the duration is not enough for the simulation to be completed. However, 0.1 $\mathrm{sec}$ and $0.01 \mathrm{sec}$ give similar results and are considered to better simulate a real crash condition.

For each crash simulation, the total deformation $(\mathrm{mm})$ and the von-Mises stress (MPa) of the helmet are examined. In Figure 5, the total deformation and the von-Mises stresses allocation is shown for the front impact for "Pacman". 


\subsubsection{Results}

As mentioned above, these analyses aim to test the durability of each concept. Therefore, the stress formed in each impact simulation must remain between the yield stress limits of plastic ABS which is 18.5 $\mathrm{MPa}-51 \mathrm{MPa}$. To better compare the results, it is important to consider the total deformation rather than the stress in order to avoid errors introduced at stress concentration areas.

In view of the foregoing, Table 1 shows both results for each concept in four different impact situations. For the "Hood" front part in rear type of impact and rear part in front type of impact, the simulation didn't converge due to the complexity of the geometric model. The concept with discontinuity on the chin area ("Elytra"), behaves worse in the rear and vertical crashes while the concept with chin protection and discontinuity at the lower back ("Pacman") behaves worse in rear crashes. Finally, the concept with chin protection and discontinuity between front and rear areas ("Hood") behaves worse in front and lateral crashes. "Pacman" presents lower deformations for higher stresses in rear, lateral and vertical impacts compared to "Elytra" and is overall better than "Hood".

Table 1. Crash simulation results

\begin{tabular}{|l|c|c|c|c|c|c|c|c|}
\hline \multirow{2}{*}{ Concept } & \multicolumn{4}{|c|}{ Total Deformation (mm) } & \multicolumn{4}{c|}{ Von Mises Stress (MPa) } \\
\cline { 2 - 10 } & \multicolumn{4}{|c|}{ Type of Impact } & \multicolumn{4}{c|}{ Type of Impact } \\
\cline { 2 - 10 } & Front & Rear & Lateral & Vertical & Front & Rear & Lateral & Vertical \\
\hline Elytra & 5.45 & 20.69 & 38.36 & 14.25 & 58.56 & 36.85 & 23.89 & 25.67 \\
\hline Pacman & 19.30 & 22.26 & 22.22 & 11.62 & 31.85 & 48.72 & 32.81 & 67.10 \\
\hline Hood (front) & 30.75 & n/a & 31.70 & 30.57 & 45.86 & n/a & 32.65 & 22.67 \\
\hline Hood (rear) & n/a & 10.07 & 19.09 & 18.58 & n/a & 45.34 & 43.75 & 34.66 \\
\hline
\end{tabular}

\subsection{Evaluation phase 2: Usability testing}

A usability testing is conducted aiming to identify potential issues regarding the ease of helmet placement and removal of each design concept. To this end, the functional models of the three design concepts were used by five experienced riders aiming to: (i) examine how well a particular concept fits the action capabilities of the user, and (ii) observe upper-body posture while performing task.

\subsubsection{Participants}

Five experienced motorcycle riders ( 2 women, 3 men), ranged in age from 40-55, volunteered to participate in the study. All participants hold a motorcycle license for more than 20 years and are all active motorcycle-riders. An overview of the participants' profiles as well as the type of motorcycle and helmet used while riding in urban traffic environment is presented in Table 2. Participants were recruited from a motorcycle club in Athens, Greece.

Table 2. Profile of the five riders participated in the usability study

\begin{tabular}{|c|c|c|c|c|c|c|}
\hline User & Sex & Age & \multicolumn{2}{|c|}{ Licence holding/ Mileage } & \multirow{2}{*}{ Motorcycle } & \multirow{2}{*}{ Helmet } \\
\cline { 4 - 5 } & & & Years & Kms/years & & \\
\hline 1 & $\mathrm{M}$ & 46 & 27 & 6000 & Dual-sport & Open-face \\
\hline 2 & $\mathrm{M}$ & 55 & 34 & 15000 & Dual-sport & Full-face \\
\hline 3 & $\mathrm{M}$ & 48 & 21 & 10000 & Scooter & Open-face \\
\hline 4 & $\mathrm{~F}$ & 40 & 24 & 6000 & Scooter & Open-face \\
\hline 5 & $\mathrm{~F}$ & 42 & 24 & 5000 & Scooter & Open-face \\
\hline
\end{tabular}

\subsubsection{Apparatus and procedure}

Participants carried out the study procedure individually. After completing their demographic information, the participants were introduced to the purpose of the study along with a brief demonstration of the three functional models corresponding to each design concept (see section 2.4). All participants were first-users of the functional models and were asked to place and then remove each helmet up to four times, while being video recorded. The participants were instructed to try -if it was necessary- different ways to place the helmet on their head, in order to make the whole process more convenient for them. The order of the prototypes used by each participant was random. After each placement/removal the participants were asked to lower their hands. The entire process lasted about 20 minutes per participant. 


\subsubsection{Data extraction}

Video-recordings are analysed by emphasizing on the design affordances that each concept provides to the user in order to facilitate the process of helmet placement and removal. Since each concept has its own way to be placed, it is necessary to determine the factors affecting the head passage during helmet placement considering the widest areas of the head, namely sagittal arc, coronal arc and maximum head diameter (Pheasant, 1990). According to this, three passing-points are designated: (a) the sagittal arc, i.e. the crown of the head, (b) the coronal arc, i.e. from ear to ear across the crown of the head, and (c) the maximum head diameter, i.e. from the chin to the most distant point on the back of the head. That way, comparison among the three concepts is made possible by considering the coupling of each design concept with the user's action capabilities while passing his/her head across these reference points. For reasons of space, only the head passage during helmet placement is presented in the next section, since the helmet removal is considered as the same task in the reverse order.

\subsubsection{Observations}

The movable frontal area of the "Elytra" contributes to an almost unimpeded helmet passage across all three passing-points (Figure 6), as a combined result of: (i) the user's neck flexion due to the opening in the front part of the helmet, facilitating the helmet passage over the sagittal arc, and (ii) the symmetric use of both hands due to the position of the helmet's lower parts, facilitating both the opening of helmet aperture and the helmet passage over the coronal arc, while the ears remain flat against the user's head.

It is worth to mention here that although the helmet passage over the maximum head diameter is virtually unimpeded, for all five participants, the act of closing the frontal area of the helmet is observed to be the most demanding and time-consuming task. As a result, the mean time duration for the helmet placement over the third passing point $(M=2579 \mathrm{~ms}, S D=287)$ is longer than the corresponding time in the previous two passing points $(M=1336 \mathrm{~ms}, S D=115$ and $M=1003 \mathrm{~ms}, S D=85$ for passing points a and $b$, respectively). A main reason for this is the side position of the retention system causing a lateral neck flexion and an imprecise movement towards the target.

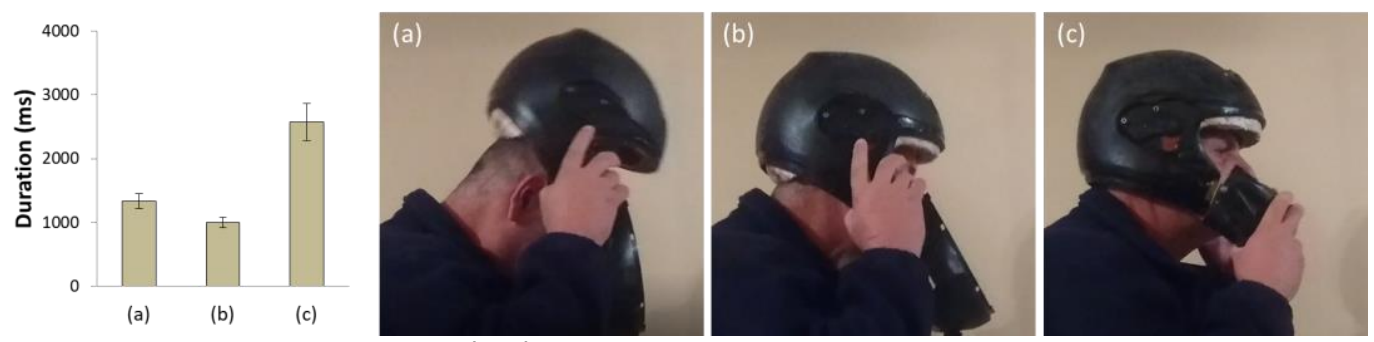

Figure 6. Mean time duration (ms) and typical body movements while a user passes the "Elytra" over his/her (a) sagittal arc, (b) coronal arc and (c) maximum head diameter.

Accordingly, the movable lower and upper areas of the "Pacman" enable a different coupling between the user and the helmet's design features (Figure 7). As it is seen in Figure 7, the helmet passage over the sagittal arc and the maximum head diameter is accomplished as the combined result of: (i) the asymmetric use of both hands -above the user's head- creating an aperture between the lower and the upper part of the helmet, and (ii) the user's neck flexion, facilitating the passage of the upper part of the helmet over the sagittal arc and the maximum head diameter, at the same time. In addition, the new position of the helmet (Figure 7b) enables (iii) the symmetric use of both hands to roll the lower part of the helmet onto the head, facilitating the helmet passage over the coronal arc whereas the ears remain flat against the user's head.

It is interesting to note that this process, although time-saving, turns the problem of head passing through the helmet aperture into a problem of targeting a new aperture (created between the lower and the upper part of the helmet) above the user's head. As a result, all five participants had a difficulty to place appropriately the two helmet areas above their heads, which is also evident in the longer mean time spent for passing the helmet over the first point $(M=1512 \mathrm{~ms}, S D=323)$ compared to the subsequent two passing points $(M=1150 \mathrm{~ms}, S D=76$ and $M=1182 \mathrm{~ms}, S D=118$ for passing points $\mathrm{b}$ and $\mathrm{c}$, respectively). In one extreme case (user \#5) a participant fully stretched the two helmet parts and then attempted to place first the lower part on her neck. This resulted to a rather abrupt lowering of the upper helmet part causing a small injury on her ears. Thus, the particular body posture with both 
hands above the shoulder joints (Figure 7a) may be a source of discomfort and possibly a source of erroneous or even accidental performance.
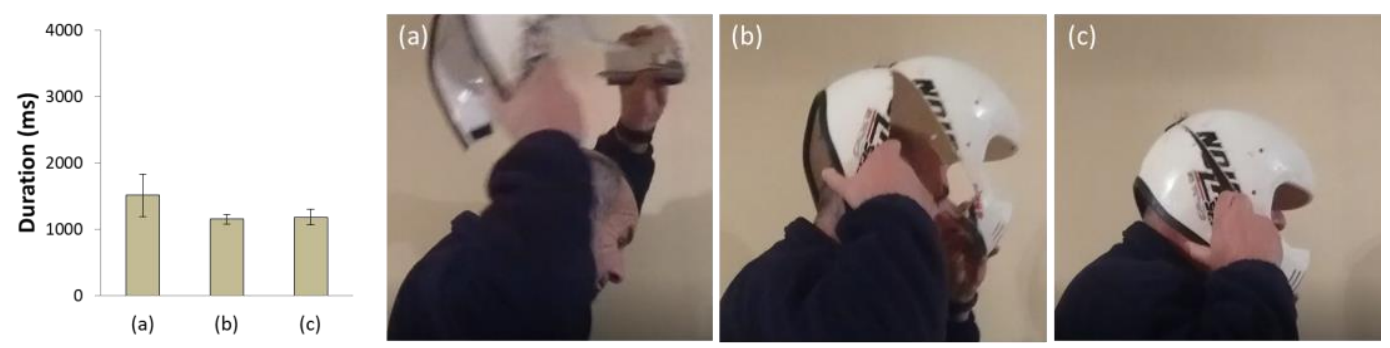

Figure 7. Mean time duration (ms) and typical body movements while a user passes the "Pacman" over his/her (a) sagittal arc, (b) maximum head diameter and (c) coronal arc.

Finally, the moving front area of the "Hood" contributes to an unimpeded head passage over the sagittal arc and the coronal arc (Figure 8), similarly to the "Elytra". The only difference between the two concepts lies in the user's hand position, i.e. both hands are placed at the lower part of the "Elytra", whereas at the upper part of the "Hood". This difference is due to the smaller surface area at the lower part of the "Hood" which, in turn, makes it easier for a user to roll the helmet onto his/her head -while she/he is holding the upper part of it- as it is seen in Figures $8 \mathrm{a}$ and $8 \mathrm{~b}$.

As for the helmet passage over the maximum head diameter (Figure 8c), a rather unexpected time delay is observed resulting int an increased mean time duration for the helmet placement over the third passing point $(M=2725 \mathrm{~ms}, S D=662)$ compared to the previous two passing points $(M=1178 \mathrm{~ms}$, $S D=207$ and $M=1004 \mathrm{~ms}, S D=275$ for passing points a and b, respectively). This time delay is partly due to the difficulty of users to pass the lower part of the helmet under their chin and partly due to imperfections of the rotation mechanism used in functional model. Nevertheless, even if the act of closing the front part of the helmet was less time-consuming, the helmet passage over the maximum head diameter would still be a source of discomfort, especially in the chin area of users.
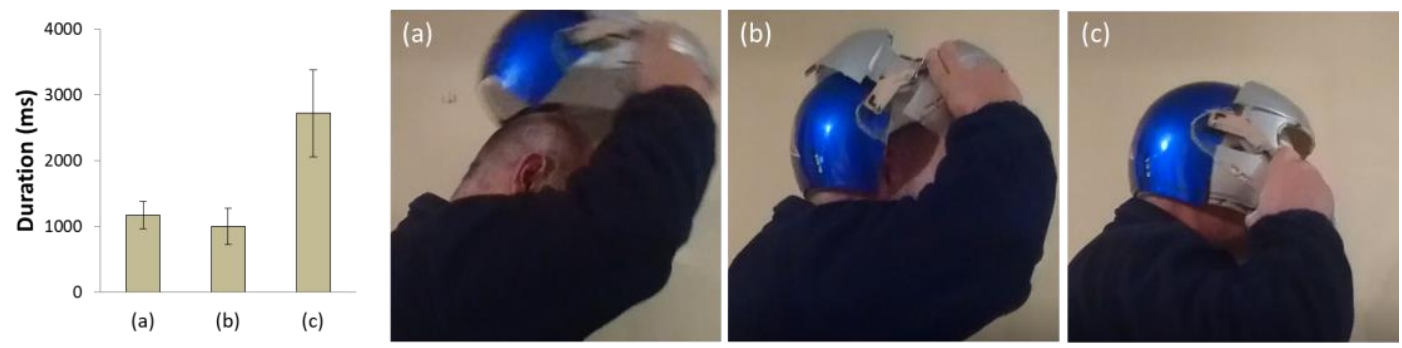

Figure 8. Mean time duration (ms) and typical body movements while a user passes the "Hood" over his/her (a) sagittal arc, (b) coronal arc and (c) maximum head diameter.

\section{Discussion}

In this study, the re-design of a motorcycle helmet for use in urban traffic environment is examined by introducing a certain design feature in the shell form (i.e. a solid body under the chin) to prevent users bypassing the strap fastening system. According to previous studies, helmeted riders in urban traffic tend to improperly secure the helmet retention system (Freitas et al, 2018; Sung et al, 2016; Ramli and Oxley, 2016; Li et al, 2008; Kulanthayan et al, 2001) offsetting, in practice, the potential benefits of using a helmet in the event of a crash. Considering the riders' practices in urban traffic, an exploration of design alternatives to eliminate the need of the typical strap fastening system poses an interesting design challenge that, if achieved, can both maximize a rider's head protection and the effectiveness of traffic enforcement.

Towards this direction, the problem-solving methodology (Dieter and Schmidt, 2013) is used to the development of three design concepts, named as "Elytra", "Pacman" and "Hood", satisfying the following design criteria: (i) helmet protection to the entirety of the head, (ii) maximum possible helmet resistance in a crash, (iii) ease of helmet placement/removal, and (iv) helmet weight less than 2000gr. All three concepts meet the first and the fourth criterion. The other two design criteria are 
evaluated by using 3D models of the three concepts in the crash simulations tests and functional prototypes of the three concepts in the usability tests, respectively.

The outcomes of this two-phase evaluation offer us some interesting insights about the pros and cons of each concept. On the one hand, results of crash simulations show that "Pacman" presents lower deformations for higher stresses in rear, lateral and vertical impacts compared to "Elytra" and it is overall better than "Hood". Also, results of usability tests showed that the mean time of wearing "Pacman" is almost one second faster than the other two concepts. On the other hand, observations of users' trials showed that "Pacman" enhances users to alter the structure of the task (i.e. helmet placement), turning the problem of head passing through the helmet aperture into a problem of targeting to a new aperture (created between the lower and the upper part of the helmet) above the user's head. A user's attempt to mentally simulate the appropriate helmet aperture above their head having as reference their upper limbs can become tricky. Due to the rotating action of the shoulder joints, alignment of a user's hands to the midline of their body is neither perfect nor relaxing. As a result, a large variation is observed in the time that users spent for placing appropriately the two helmet parts above their head. In contrast, wearing "Elytra" or "Hood" turns out to be cumbersome and time consuming only during the act of closing the helmet.

In the light of these observations, it becomes clear that all three concepts need further development to eliminate awkward body postures and time delays. However, given the crash simulation results, "Pacman" seems to be the most promising concept both in terms of safety and ease of placement. To this end, further development of design features is needed for aiding a user to appropriately adjust the helmet aperture and to roll the helmet over their head, e.g. through improving tactile feedback.

Another major issue mentioned by the participants while overviewing the three concepts, is a sense of insecurity caused by the lack of a typical strap fastening system. Their main concern is related to the potential failure(s) of the locking mechanism(s) in the case of a crash, which they consider much more likely to occur due to wear of the locking mechanism compared to a strap fastening system. Evidently, elimination of failures of the locking mechanism is an issue of outmost importance that needs to be examined in future studies.

To enhance a reader to evaluate the above findings, a few limitations of this study are worth mentioning. Firstly, the results are based on only five participants suggesting that their generalization needs to be verified in further studies. Secondly, the prototypes used in this study are all the same size and lacked in-house comfort paddings providing at least some participants with ill-fitting designs. Thus, the mean times reported in this study should not be taken into account nominally but only comparatively among the three concepts. Finally, the reported crash simulation results are limited to particular surface conditions. In future work, redesign of the surface conditions on the 3D models can be implemented so as to re-evaluate the impact simulations and the high stress values observed locally.

\section{CONCLUSIONS}

In this paper, the re-design approach of an urban motorcycle helmet to prevent users bypassing the strap fastening system is presented. Three helmet design concepts are developed and evaluated using impact simulations and usability tests. A helmet design with solid body under the chin, as means for enhancing proper use of helmet in urban traffic, can negatively affect the ease of placement as well as the helmet resistance in the event of a crash. To tackle these two issues, a helmet form with two solid bodies intersected diagonally on the horizontal axis, i.e. "Pacman", was found to be the most promising design approach both in terms of safety and speed of helmet placement. Further improvement of the helmet design features towards enhancing a user to appropriately adjust the helmet aperture over their head (e.g. via tactile feedback) is found as necessary, in order to eliminate awkward body postures and time delays. Also, ensuring elimination of failures of the locking mechanism is an issue of importance that needs to be examined. Future studies should focus on the development of an improved prototype towards the acceptability of the product.

\section{REFERENCES}

Dieter, G.E. and Schmidt, L.C. (2013), Engineering Design, McGraw-Hill, New York.

Esmaeilikia, M., Radun, I., Grzebieta, R. and Olivier, J. (2019), "Bicycle helmets and risky behaviour: A systematic review", Transportation Research Part F: Traffic Psychology and Behaviour, Vol. 60, pp. 299-310. https://doi.org/10.1016/j.trf.2018.10.026 
Freitas, C.A., Furtado, A.M., Petterle, R.R., Pimentel, S.K. and Carvalho, F.H. (2018), "Helmet retention system types as a risk factor to properly attach the chin strap", Traffic Injury Prevention, Vol. 19 No. 2, pp. 173-178. https://doi.org/10.1080/15389588.2017.1356022

Hung, D.V., Stevenson, M.R. and Ivers, R.Q. (2008), "Barriers to, and factors associated, with observed motorcycle helmet use in Vietnam”, Accident Analysis and Prevention, Vol. 40 No. 4, pp. 1627-1633. https://doi.org/10.1016/j.aap.2008.05.002

Jung, S., Xiao, Q. and Yoon, Y. (2013), "Evaluation of motorcycle safety strategies using the severity of injuries", Accident Analysis and Prevention, Vol. 59, pp. 357-364. https://doi.org/10.1016/j.aap.2013.06.030

Khan, I., Khan, A., Aziz, F., Islam, M. and Shafqat, S. (2008), "Factors associated with helmet use among motorcycle users in Karachi, Pakistan”, Academic Emergency Medicine, Vol. 15 No. 4, pp. 384-387. https://doi.org/10.1111/j.1553-2712.2008.00049.x

Kim, S., Ro, Y.S., Shin, S.D., Song, K.J., Hong, K.J. and Jeong, J. (2018), "Preventive effects of motorcycle helmets on intracranial injury and mortality from severe road traffic injuries", American Journal of Emergency Medicine, Vol. 36 No. 2, pp. 173-178. https://doi.org/10.1016/j.ajem.2017.07.044

Kulanthayan, S., Umar, R.S., Hariza, H.A. and Nasir, M.T. (2001), "Modeling of compliance behavior of motorcyclists to proper usage of safety helmets in Malaysia", Journal of Crash Prevention and Injury Control, Vol. 2 No. 3, pp. 239-246. https://doi.org/10.1080/10286580108902568

Li, L.P., Li, G.L., Cai, Q.E., Zhang, A.L. and Lo, S.K. (2008), "Improper motorcycle helmet use in provincial areas of a developing country", Accident Analysis and Prevention, Vol. 40 No. 6, pp. 1937-1942. https://doi.org/10.1016/j.aap.2008.06.019

Lin, M.R. and Kraus, J.F. (2009), "A review of risk factors and patterns of motorcycle injuries", Accident Analysis and Prevention, Vol. 41 No. 4, pp. 710-722. https://doi.org/10.1016/j.aap.2009.03.010

Otte, D. (1991), "Technical demands on safety in the design of crash helmets for biomechanical analysis of real accident situations", SAE Technical Paper, No. 912911, p. 15. https://doi.org/10.4271/912911

Ouellet, J.V. (2011), "Helmet use and risk compensation in motorcycle accidents", Traffic Injury Prevention, Vol. 12 No. 1, pp. 71-81. https://doi.org/10.1080/15389588.2010.529974

Pahl, G., Beitz, W., Feldhusen, J. and Grote, K.-H. (2007). Engineering design: A systematic approach, Springer-Verlag, London.

Pheasant, S. (1990), Anthropometrics: An Introduction, British Standards Institution, London.

Ramli, R., Oxley, J., Hillard, P., Mohd Sadullah, A.F. and McClure, R. (2014), "The effect of motorcycle helmet type, components and fixation status on facial injury in Klang Valley, Malaysia: A case control study", BMC Emergency Medicine, Vol. 14, p. 17. https://doi.org/10.1186/1471-227X-14-17

Ramli, R. and Oxley, J. (2016), "Motorcycle helmet fixation status is more crucial than helmet type in providing protection to the head", Injury, Vol. 47 No. 11, pp. 2442-2449. http://dx.doi.org/10.1016/j.injury.2016.09.022

Saunders, R.N., Witte, A.B., Adams, N.S., Dull, M.B., Davis, A.T., Gibson, C.J., Iskander, G.A., Rodriguez, C.H., Cohle, S.D. and Chapman, A.J. (2020), "Does the repeal of mandatory motorcycle safety legislation affect the deaths of motorcyclists? An autopsy-based study evaluating the impact of Michigan's universal helmet law repeal on immediately fatal motorcycle crashes", The American Surgeon, Vol. 86 No. 4, pp. 205-207. https://doi.org/10.1177/000313482008600415

Skalkidou, A., Petridou, E., Papadopoulos, F.C., Dessypris, N. and Trichopoulos, D. (1999), "Factors affecting motorcycle helmet use in the population of greater Athens, Greece", Injury Prevention, Vol. 5 No. 4 , pp. 264-267. http://dx.doi.org/10.1136/ip.5.4.264

Suh, N.P. (1990), The principles of design, Oxford University Press, New York.

Sung, K.M., Noble, J., Kim, S.C., Jeon, H.J., Kim, J.Y., Do, H.H., Park, S.O., Lee, K.R. and Baek, K.J. (2016), "The preventive effect of head injury by helmet type in motorcycle crashes: A rural Korean single-center observational study", BioMed Research International, Vol. 2016, p. 1849134. https://doi.org/10.1155/2016/1849134

Thai, K.T., McIntosh, A.S. and Pang, T.Y. (2015), "Factors affecting motorcycle helmet use: Size selection, stability, and position", Traffic Injury Prevention, Vol. 16 No. 3, pp. 276-282. https://doi.org/10.1080/15389588.2014.934366

Tsai, Y.J., Wang, J.D. and Huang, W.F. (1995), "Case-control study of the effectiveness of different types of helmets for the prevention of head injuries among motorcycle riders in Taipei, Taiwan", American Journal of Epidemiology, Vol. 142 No. 9, pp. 974-981. https://doi.org/10.1093/oxfordjournals.aje.a117746

Ullman, D.G. (2002), The mechanical design process, McGraw Hill, New York

World Health Organization (2018), Global status report on road safety 2018, World Health Organization, Geneva.

Yu, W.Y., Chen, C.Y., Chiu, W.T. and Lin, M.R. (2011), "Effectiveness of different types of motorcycle helmets and effects of their improper use on head injuries", International Journal of Epidemiology, Vol. 40 No. 3, pp. 794-803. https://doi.org/10.1093/ije/dyr040 